\title{
Cuidados de Enfermería dirigidos a una paciente con Insuficiencia Venosa Crónica. A propósito de un caso
}

\author{
Antonia M. Pérez Roca \\ Graduada en Enfermería. Trabajadora independiente. España \\ Correspondencia \\ Antonia M. Pérez Roca \\ Correo electrónico: aperezroca23@gmail.com \\ (Recibido: 29/03/2018. Aceptado: 11/05/2018)
}

\begin{abstract}
RESUMEN
La Insuficiencia Venosa Crónica es una patología progresiva, la cual es más prevalente entre el sexo femenino. Ciertos estudios demuestran que la enfermedad venosa altera la movilidad, el sueño, provoca efecto psicológico negativo y una disminución de la calidad de vida del paciente. Se presenta el caso clínico de una paciente de 65 años con diversas patologías, entre ellas Insuficiencia Venosa Cónica, que presenta baja adherencia terapéutica, sobrepeso, problemas de autoimagen, disconfort y déficit de conocimientos. El objetivo planteado fue proporcionar cuidados enfermeros que aporten al paciente conocimientos sobre su enfermedad, aumenten la adherencia terapéutica a su tratamiento y mejoren su autoimagen y confort. Gracias a una adecuada planificación de cuidados, la paciente expresó gran satisfacción tras ampliar sus conocimientos y habilidades de autocuidado. Además presentó cambios significativos en su piel, mostró mejoría en su descanso y mayor aceptación hacia su imagen. Como conclusión, la educación para la salud en el paciente crónico es esencial para lograr un adecuado automanejo de su enfermedad y, así, aumentar la satisfacción con su proceso de salud.
\end{abstract}

\section{PALABRAS CLAVE}

Insuficiencia Venosa; Cuidados de Enfermería; Educación en Salud

\section{SUMMARY}

Chronic Venous Insufficiency is a progressive pathology, which is more prevalent among females. Some studies show that venous disease impairs mobility, sleep, causes negative psychological effect and decrease the patient's quality of life. We present a clinical case of a 65 -year-old patient with various pathologies, including chronic venous insufficiency, which presents low therapeutic adherence, overweight, self-image problems, discomfort and knowledge deficit.

The aim was to provide nursing care that gives knowledge to the patient about his illness, increases the therapeutic adherence to his treatment and improves his self-image and comfort. Thanks to the proper care planning, the patient expressed great satisfaction after expanding her knowledge and self-care skills. He also presented significant changes in his skin, showed improvement in his rest and greater acceptance of his image.

In conclusion, education for chronic patient health is essential to achieve an adequate self-management of their disease and, thus, increase satisfaction with their health process.

\section{KEYWORDS}

Venous insufficiency; Nursing Care; Health Education

\section{Introducción}

La Insuficiencia Venosa Crónica (IVC) es una patología progresiva y se distingue como una de las enfermedades de mayor morbilidad. Su prevalencia aproximada es del 20 al $50 \%$ en la población femenina y del 10 al $20 \%$ de la población masculina. La IVC es una alteración funcional del retorno venoso, debido a una insuficiencia o incompetencia valvular venosa con o sin obstrucción venosa. Diferentes síntomas pueden aparecer en los primeros estadios como son: dolor, pesadez, calambres nocturnos, tumefacción y prurito en los miembros inferiores (1).
En el estudio de Delia Charles Edouard et al (1), el $74,5 \%$ de los enfermos con patología venosa eran mujeres. Se destacaron, como factores de riesgo, profesiones que exigen poca movilidad, largos períodos en posición ortostática y un tiempo corto de reposo. Además, el sobrepeso y la vida sedentaria, ya que ambas permiten que aumente la sobrecarga de peso a nivel de las extremidades inferiores; antecedentes familiares; tabaquismo; multiparidad $(1,2)$; el estreñimiento y una dieta pobre en fibras (2). Además, se relacionó la pérdida de movilidad, efecto psicológico negativo y disminución de la calidad de 
vida con la enfermedad venosa (3). Sin embargo, Farias et al (3) destacaron que la alteración del patrón del sueño se relaciona con el dolor en pacientes con IVC, aunque su estudio reflejó que el $77 \%$ de los pacientes afirmaron dormir un número de horas de sueño satisfactorias.

Según Bellmunt et al (4), la prevalencia de consultas por enfermedad vascular en Atención Primaria representa el 3,4\% del total de las consultas, constituyendo las flebopatías la primera causa (69\%), y en este grupo, las varices suponen la mitad de los casos (30,2\%).

Silva- Pupo et al (2), refieren que las varices sintomáticas disminuyen de forma considerable la calidad de vida, sobre todo en la dimensión física y del dolor. Se destaca la inclusión de medidas higiénico-dietéticas para el tratamiento de las varices, que consisten en control del peso, ejercicio y la terapia postural, incidiendo en la terapia compresiva para la disminución de la hipertensión venosa, la cual favorece el desarrollo y la progresión de la enfermedad.

El objetivo de este estudio es proporcionar, a una paciente con IVC, cuidados enfermeros que le aporten conocimientos sobre su enfermedad y aumenten la adherencia terapéutica a su tratamiento, autoimagen y confort.

\section{Método}

Se ha diseñado un estudio observacional, descriptivo, tipo estudio de caso. Dicho estudio se ha realizado entre los meses de diciembre 2017 y marzo de 2018 para la fase de contacto, observación del paciente y recogida de los datos clínicos. La paciente otorgó su consentimiento para la participación en el estudio, y a lo largo del mismo se ha respetado su anonimato y la confidencialidad de los datos según las normas vigentes (LOPD 15/1999 y Ley 41/2002).

Se realizó una búsqueda bibliográfica en Pubmed y Scielo, desarrollada a partir de los Descriptores de las Ciencias de la Salud (DeCS): Insuficiencia Venosa, Atención de Enfermería, Educación en Salud. Se revisaron artículos publicados entre $2010-2018$, con acceso completo, en español y/o inglés.

\section{Caso clínico}

Se presenta el caso clínico de una mujer de 67 años de edad, obesa, con incontinencia urinaria y con Insuficiencia Venosa, que refiere insomnio e insatisfacción con su estado de salud.

Se elaboró un Plan de Cuidados en el que la valoración se realizó a partir de los patrones funcionales de M. Gordon (5). Para formular los diagnósticos de enfermería (DxE), los criterios de resultados y las intervenciones enfermeras se utilizaron taxonomías enfermeras: la NANDA Internacional 2015-2017 (6), la Clasificación de Resultados de Enfermería (NOC) (7) y la Clasificación de Intervenciones de Enfermería NIC (8), respectivamente.

\section{Valoración enfermera}

Patrón no 1 Manejo de la Salud

- No refiere hábitos tóxicos.

- Refiere conocer su proceso de salud, aunque destaca escasos conocimientos.

- Test de Morisky-Green: buena adherencia al tratamiento farmacológico y medias compresivas.

- No alergias a medicamentos

- Antecedentes personales: HTA; Ictus; IVC; Obesidad; DLP; Pie plano; Tiroides multimicronodular; Liquen plano; STC; Depresión; Vértigos.

- Antecedentes familiares: Madre: IAM; Padre: neoplasia de colon; Hermano: DMII.

- Tratamiento: Ranitidina hidrocloruro 300 mg. Amitriptilina hidrocloruro $10 \mathrm{mg}$. Atorvastatina $40 \mathrm{mg}$. Clopidogrel $75 \mathrm{mg}$. Buspirona $10 \mathrm{mg}$. Troxerutina Polvo sabor naranja $1000 \mathrm{mg}$. Urea $30 \%$ tópico. Betametasona (dipropionato) y ácido salicílico.

Patrón no 2 Nutricional-Metabólico

- Obesidad. IMC 30,81 kg/m2.

- DLP.

- Refiere realizar dieta mediterránea. Refiere picotear demasiado.

- Estado de la piel deshidratado. Hiperqueratosis plantar. No signos infección. No rubor. No dolor a la palpación. Prurito ocasional. Fig. 1

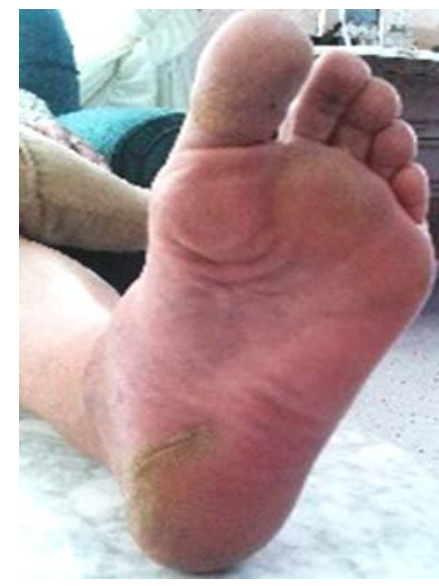

Fig. 1. Pie izquierdo hiperqueratosis plantar 15/12/2017

- Refiere cansancio MMII. No claudicación intermitente. Pulso pedio y tibial conservados.

- Venas varicosas y reticulares ambos MMII. Escleroterapia en 2004-2006. Fig. 2

- Clasificación IVC (CEAP): C3: Edema. Grado 1: Depresión leve / Desaparición casi instantánea.

Patrón no 3 De Eliminación

- Incontinencia urinaria de esfuerzo.

- Estreñimiento ocasional.

Patrón no 4 De Actividad - Ejercicio

- Tensión Arterial: 130/70 mmHg. Frecuencia cardiaca: 78 lpm.

- Refiere caminar todos los días. 


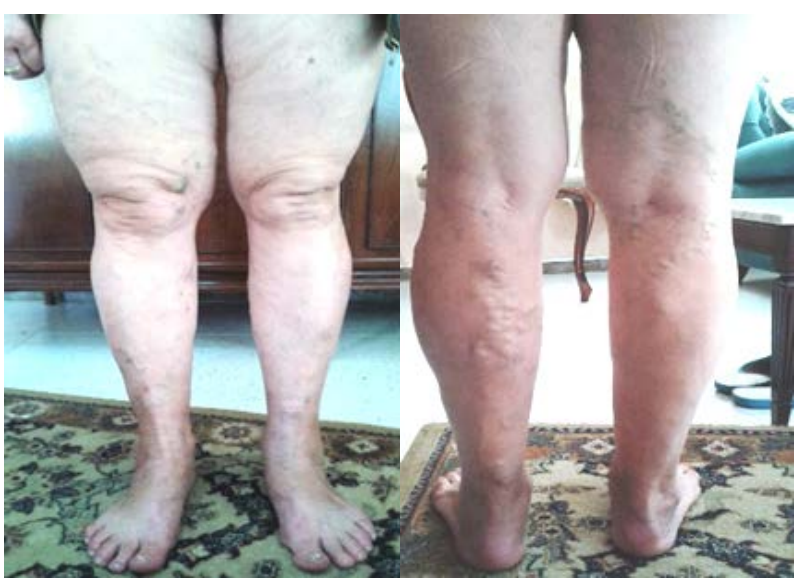

Fig. 2. Vista anterior y posterior MMII 15/12/2017.

Patrón no 5 De Reposo-Sueño

- Refiere no descansar.
Patrón no 6 Cognitivo-Perceptual

- Paciente orientada y consciente.

- No refiere dolor (EVA 0).

Patrón no 7 Autopercepcion-Autoconcepto

- Refiere "no gustarle sus piernas", "desde joven he tenido que llevar pantalón para que no me vieran las varices".

Patrón no 8 Rol-Relaciones

- Casada.

- Jubilada.

- Relaciones adecuadas.

Patrón no 9 Sexualidad-Reproducción

- Multípara. 3 varones.

Patrón no 10 Adaptación-Tolerancia al Estrés

- La paciente refiere adaptarse a su situación de salud.

Patrón no 11 Creencias

- La religión juega un papel importante en su vida.

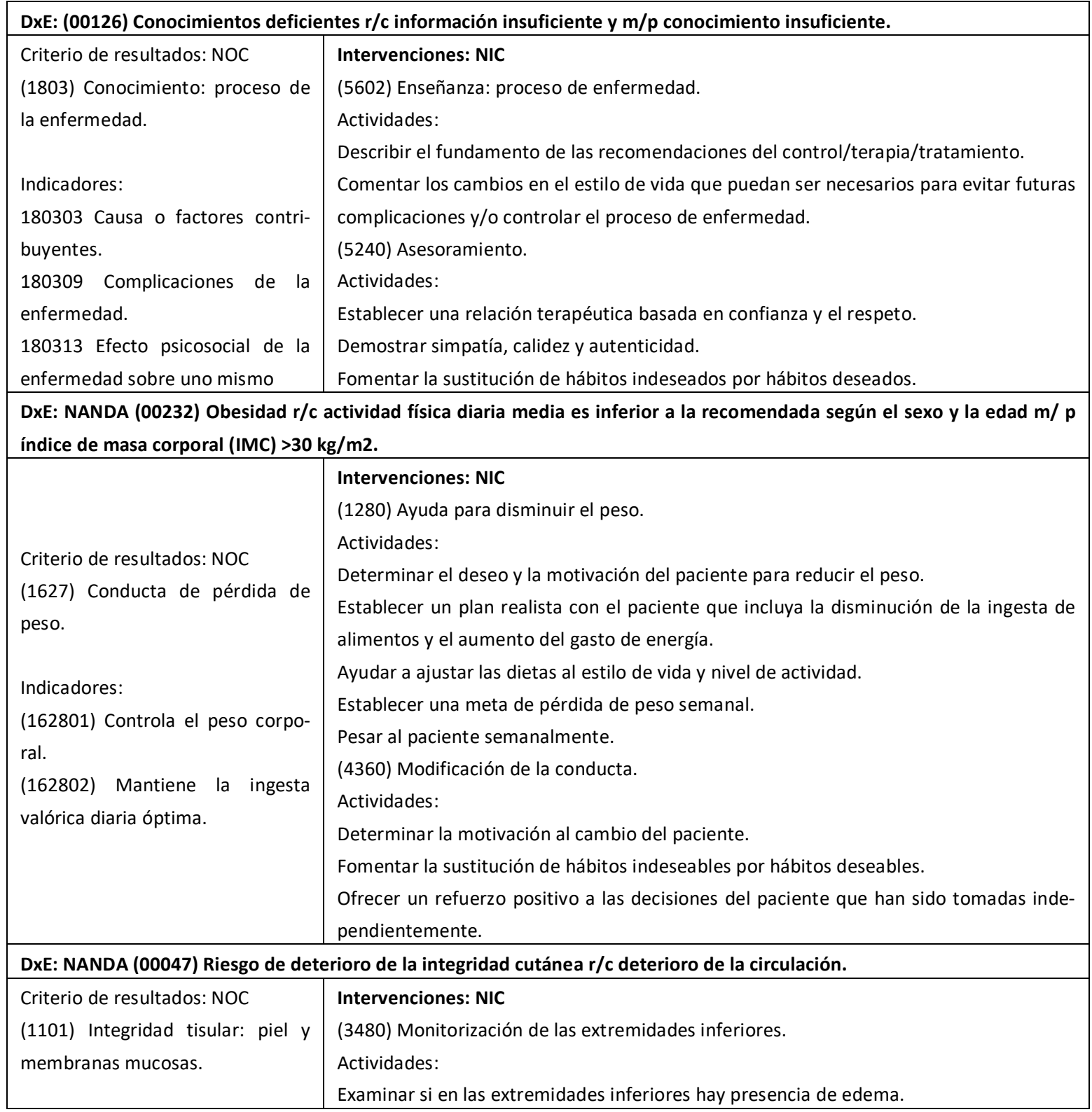




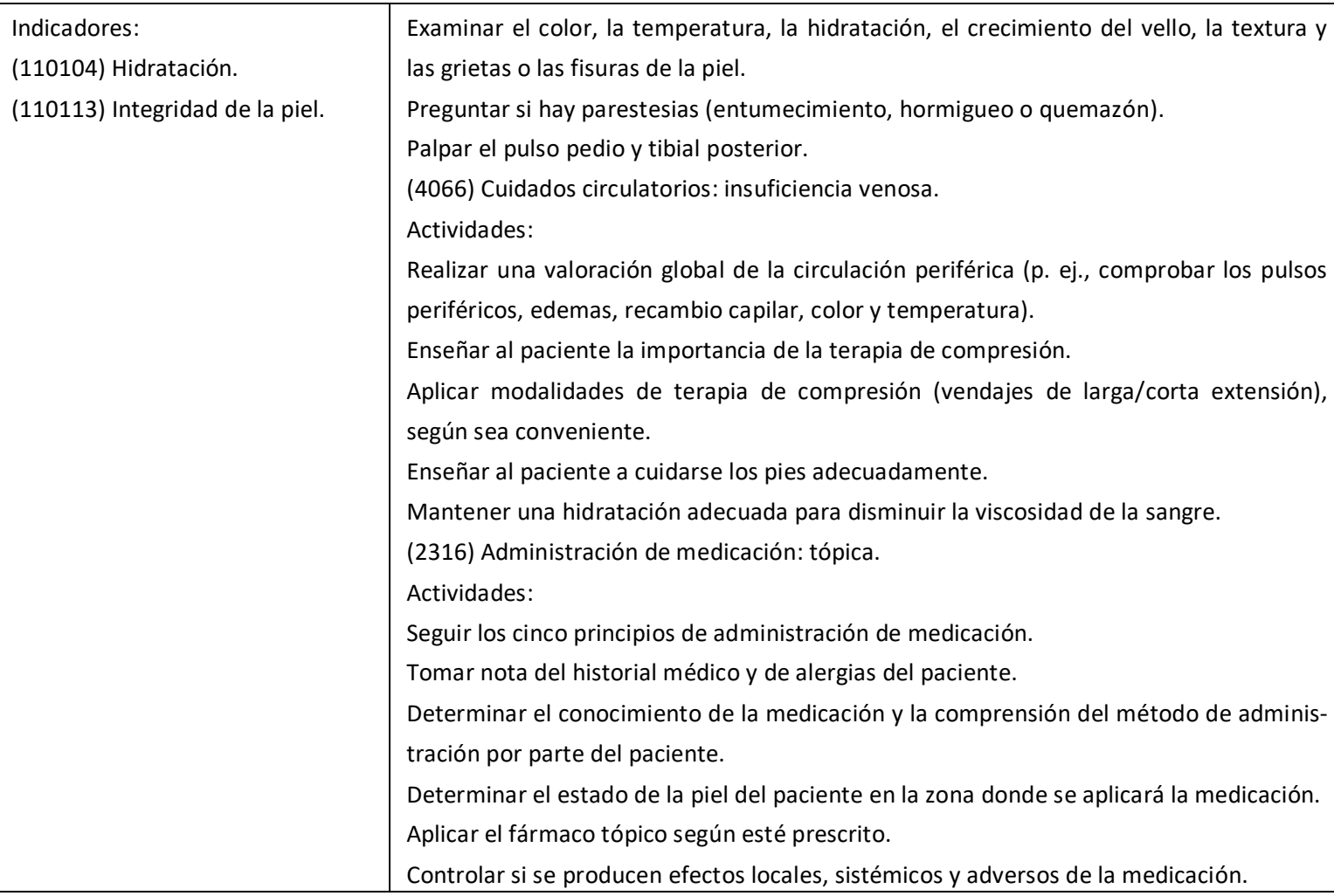

Determinar el estado de la piel del paciente en la zona donde se aplicará la medicación.

Aplicar el fármaco tópico según esté prescrito.

Controlar si se producen efectos locales, sistémicos y adversos de la medicación.

DxE: NANDA (00017) Incontinencia urinaria de esfuerzo $\mathrm{r} / \mathrm{c}$ cambios degenerativos en los músculos pélvicos $\mathrm{m} / \mathrm{p}$ observación de pérdida involuntaria de pequeñas cantidades de orina con el esfuerzo

Criterio de resultados: NOC. (0502) Continencia Urinaria.

Indicadores:

050208- Capaz de comenzar e interrumpir el chorro de la orina. 050211- Pérdidas de orina al aumentar la presión abdominal (estornudar, reír, levantar pesos).

Criterio de resultados: NOC. (0004) Descanso.

Indicadores:

000401- Horas de sueño. 000404- Calidad del sueño. 000418- Duerme toda la noche.

\section{Intervenciones: NIC.}

(0560) Ejercicio del Suelo Pélvico.

Actividades:

Enseñar al individuo a ajustar y luego relajar el anillo muscular alrededor de la uretra y del ano, como si intentara evitar orinar o el movimiento intestinal.

Enseñar a la mujer a realizar ejercicios para apretar el músculo, practicando 300 contracciones cada día, manteniendo las contracciones durante 10 segundos cada vez y descansando como mínimo 10 segundos entre contracciones, según protocolo del centro.

Explicar a la mujer que la efectividad de los ejercicios se consigue a las 6-12 semanas. (0610) Cuidados de la incontinencia urinaria.

Actividades:

Controlar periódicamente la eliminación urinaria, incluyendo la frecuencia, consistencia, olor, volumen y color.

Mostrar una respuesta positiva a cualquier disminución de los episodios de incontinencia. Limitar los líquidos durante 2 o 3 horas antes de irse a la cama.

DxE: NANDA (00096) Deprivación del sueño $\mathrm{r} / \mathrm{c}$ disconfort prolongado $\mathrm{m} / \mathrm{p}$ malestar e irritabilidad.

\section{Intervención: NIC.}

(1850) Mejorar el sueño.

Actividades:

Explicar la importancia de un sueño adecuado durante la enfermedad, las situaciones de estrés psicosocial, etc.

Controlar la ingesta de alimentación y bebidas a la hora de irse a la cama para determinar los productos que faciliten o entorpezcan el sueño.

Comprobar el esquema de sueño del paciente y observar las circunstancias físicas (apnea del sueño, vías aéreas obstruidas, dolor/molestias y frecuencia urinaria) y/o psicológicas (miedo o ansiedad) que interrumpen el sueño.

Enseñar al paciente a realizar una relajación muscular autogénica $u$ otras formas no farmacológicas de inducción del sueño. 


\begin{tabular}{|c|c|}
\hline \multicolumn{2}{|c|}{ DxE: NANDA (00118) Trastorno de la imagen corporal $r / c$ enfermedad, lesión $\mathrm{m} / \mathrm{p}$} \\
\hline $\begin{array}{l}\text { Criterio de resultados: NOC. } \\
\text { (1202) Identidad. } \\
\text { Indicadores: } \\
\text { 120213- Verbaliza confianza en sí } \\
\text { mismo. }\end{array}$ & $\begin{array}{l}\text { Intervención NIC: } \\
\text { (5270) Apoyo emocional. } \\
\text { Actividades: } \\
\text { Comentar la experiencia emocional con el paciente. } \\
\text { Realizar afirmaciones enfáticas o de apoyo. } \\
\text { Abrazar o tocar al paciente para proporcionarle apoyo. } \\
\text { Animar al paciente a que exprese sentimientos de ansiedad, o tristeza. } \\
\text { Escuchar las expresiones de sentimientos y creencias. } \\
\text { (5400) Potenciación de la autoestima. } \\
\text { Actividades: } \\
\text { Observar las frases del paciente sobre su propia valía. } \\
\text { Animar al paciente a identificar sus virtudes. } \\
\text { Reafirmar las virtudes personales que identifique el paciente. } \\
\text { Mostrar confianza en la capacidad del paciente para controlar una situación. } \\
\text { Recompensar el progreso del paciente en la consecución de objetivos. }\end{array}$ \\
\hline \multicolumn{2}{|c|}{ DxE: NANDA (00214) Disconfort r/c síntomas relacionados con la enfermedad m/p. Insatisfacción con la situación } \\
\hline $\begin{array}{l}\text { Indicadores: } \\
\text { 200801- Bienestar físico. } \\
\text { 200803- Bienestar psicológico. }\end{array}$ & $\begin{array}{l}\text { Intervención NIC: } \\
\text { (4470) Ayuda en la modificación de sí mismo. } \\
\text { Actividades: } \\
\text { Animar al paciente a examinar los valores y creencias personales y la satisfacción con } \\
\text { ellos. } \\
\text { Valorar las razones del paciente para desear cambiar. } \\
\text { Valorar el nivel de conocimiento y habilidad actual del paciente en relación con el } \\
\text { cambio deseado. }\end{array}$ \\
\hline
\end{tabular}

Tabla 1. Puntuación inicial de cada NOC obtenida mediante valoración de sus indicadores

\begin{tabular}{lccc}
\hline \multicolumn{1}{c}{ NOC } & Puntuación Inicial & Puntuación Diana & Tiempo consecución \\
\hline (1803) Conocimiento: proceso de la enfermedad. & 6 & 15 & 90 días \\
\hline (1627) Conducta de pérdida de peso. & 2 & 10 & 90 días \\
\hline (1101) Integridad tisular: piel y membranas mucosas. & 6 & 10 & 90 días \\
\hline (0502) Continencia Urinaria. & 4 & 10 & 90 días \\
\hline (0004) Descanso. & 6 & 15 & 90 días \\
\hline (1202) Identidad. & 2 & 5 & 90 días \\
\hline (2008) Estado de comodidad. & 6 & 10 & 90 días \\
\hline
\end{tabular}

Escala: 1. Gravemente comprometido. 2. Sustancialmente comprometido. 3. Moderadamente comprometido. 4. Levemente comprometido. 5. No comprometido.

Fuente: Elaboración propia basada en NOC (7).

Tabla 2. Resultados de los NOC incluidos en el Plan de Cuidados

\begin{tabular}{l|cccc}
\hline \multicolumn{1}{c|}{ NOC } & $\begin{array}{c}\text { Puntuación } \\
\text { Inicial }\end{array}$ & $\begin{array}{c}\text { Puntuación } \\
\text { Diana }\end{array}$ & $\begin{array}{c}\text { Puntuación } \\
\text { Alcanzada }\end{array}$ & $\begin{array}{c}\text { Tiempo de } \\
\text { consecución }\end{array}$ \\
\hline (1803)Conocimiento: proceso de la enfermedad. & 6 & 15 & 12 & 90 días \\
\hline (1612) Control Peso. & 2 & 10 & 6 & 90 días \\
\hline (1101) Integridad tisular: piel y membranas mucosas. & 6 & 10 & 8 & 90 días \\
\hline (0502) Continencia Urinaria. & 4 & 10 & 8 & 90 días \\
\hline (0004) Descanso. & 6 & 15 & 9 & 90 días \\
\hline (1202) Identidad. & 2 & 5 & 4 & 90 días \\
\hline (20098) Estado de comodidad. & 6 & 10 & 8 & 90 días \\
\hline
\end{tabular}

Escala: 1. Gravemente comprometido. 2. Sustancialmente comprometido. 3. Moderadamente comprometido. 4. Levemente comprometido. 5. No comprometido.

Fuente: Elaboración propia basada en NOC (7). 


\section{Valoración de los indicadores}

En la tabla 1 se observa la puntuación inicial de cada NOC obtenida mediante valoración de sus indicadores en base a Escala Likert: 1: Gravemente comprometido 2: Sustancialmente comprometido. 3: Moderadamente comprometido. 4: Ligeramente comprometido. 5: No comprometido.

\section{Resultados}

En la Tabla 2, se muestran la puntuación final obtenida de cada NOC mediante valoración de sus indicadores en base a Escala Likert.

Para el manejo etiológico de la patología vascular, se recomendó la utilización de compresión terapéutica, con medias de compresión de $21 \mathrm{mmHg}$. La paciente comentó que gracias a las medias de compresión, notó mejoría y "descanso" en sus piernas, además de una mejora en su IVC, y refirió también "tener las piernas más claras".

En las intervenciones planificadas para dar cumplimiento al NOC (1101) Integridad tisular: piel y membranas mucosas, se estableció una pauta de higiene y limpieza, mediante agua y jabón, y secando bien para desprender la queratosis y una adecuada hidratación en EEII, mediante una crema emoliente, en este caso Epaderm ${ }^{\circledR}$ por la noche.

Se obtuvo una pérdida, significativa de peso, la paciente, en su inicio, pesaba $75 \mathrm{~kg}$ y al final de la intervención pesó $69 \mathrm{~kg}$ (IMC actual: 28,35 kg/m2, sobrepeso). Esta reducción, conllevó una mejora emocional, ya que paciente refirió "que esto también le ayudó a sentirse mejor consigo misma".

Por otra parte, se realizó adiestramiento para la práctica de los ejercicios de Kegel.

\section{Discusión}

Los resultados muestran que, tras la realización de las intervenciones enfermeras, se aprecia una mejoría evidente en la situación clínica y emocional de la paciente. Cabe mencionar la aparente mejoría en las EEII de la paciente, la cual en su inicio (según imágenes 12), mostraba un déficit de hidratación e higiene y signos de IVC. Además, es destacable la adquisición de conocimientos acerca de su enfermedad y cuidados. Gracias a ello, la paciente alega mejora en sus autocuidados y en el manejo de su enfermedad.

Según Córdoba (9), el personal de enfermería debe asumir sus responsabilidades en el ámbito de la educación sanitaria dirigida a las enfermedades crónicas, que debe ser un modelo proactivo y programado de cuidados continuados centrados en el mismo paciente, y de esta manera, se conseguirán alcanzaran los objetivos.

Imbernón et al (10), argumentaron que la Enfermedad Venosa crónica, ocasiona en el paciente signos de cansancio, pesadez, debilidad, opresión, dolor y calambres, a nivel muscular de las EEII. Con el progreso de la enfermedad, pueden aparecer venas dilatadas, reticulares y varicosas, desarrollándose además, edema en los miembros, hiperpigmentación marronácea y azul- grisácea y eccema de estasis. En el caso de la paciente en estudio, se observaban venas reticulares y varicosas, además de edema leve (imágenes 1,2), registrando un CEAP 3, grado 1.

Entre las recomendaciones se incluyó la utilización de medias de compresión que la paciente aceptó bien, obteniendo un buen cumplimiento terapéutico. Este resultado se encuentra en línea con el estudio de Clark (11), que estableció que las medias de compresión proporcionan numerosos beneficios en las personas con problemas circulatorios en EEII, en comparación con la aplicación de no compresión. En nuestro caso se indicaron medias de compresión de clase I, con una presión de 20 a $30 \mathrm{mmHg}$, indicadas para venas varicosas, edema leve, o debilidad muscular, síntomas presentes en la paciente. Otros estudios $(12,13)$ también destacaron los beneficios del uso terapéutico de medias de compresión. Sin embargo, identificaron, que alrededor de las tres cuartas partes de los pacientes encuestados, rechazaban el uso de las medias de compresión indicadas. Esto se debió, a que refirieron tras su uso, "una compresión significativamente mayor que las medias y calcetines de uso corriente", demostrándose que la falta de educación de los pacientes, fue la base de la falta de cumplimiento terapéutico.

El uso cotidiano de Epaderm ${ }^{\circledR}$ mejoró la higiene e hidratación, aumentando el confort de la paciente; también se observó que, conforme pasaban las semanas, la hiperqueratosis plantar iba desapareciendo (Fig. 3). Otros autores, han obtenido resultados equivalentes a los nuestros $(10,14)$.

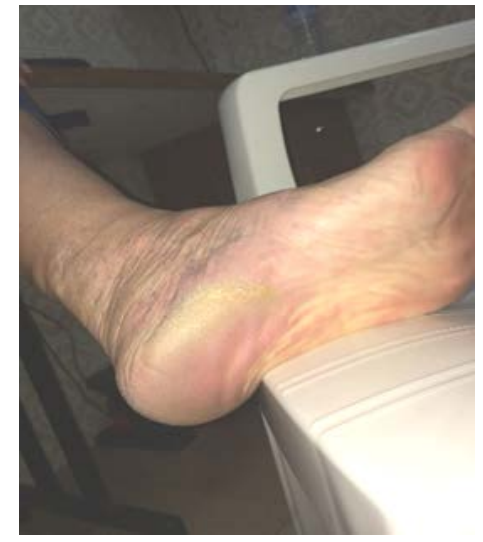

Fig. 3. Pie izquierdo hiperqueratosis planta 8/01/2018.

Destacamos que, entre los cuidados que requiere el paciente con IVC, la compresión terapéutica, la higiene y la hidratación de la piel con emolientes, son cuidados imprescindibles.

La paciente presentó una considerable reducción de peso durante el programa de intervención, lo que contribuyó de manera significativa a reducir su obesidad. Se le aconsejó realizar una dieta ligera, rica en fibra para evitar estreñimiento, debido a que este hace empeorar la insuficiencia venosa al aumentar la tensión intraabdominal (15). Los estudios poblacionales (16) muestran 
una mayor incidencia de patología vascular crónica en personas obesos o con sobrepeso importante, con un incremento del riesgo de desarrollar úlceras venosas.

También se produjo una mejora de la incontinencia urinaria tras el adiestramiento y la práctica continuada de los ejercicios de Kegel. A lo largo de los meses, mostró cierta mejoría y fortalecimiento de dicha musculatura. En la bibliografía encontramos datos similares que ponen de manifiesto que, el tratamiento de la incontinencia urinaria de esfuerzo, mediante ejercicios de entrenamiento de la musculatura del suelo pélvico, logra la mejora de los síntomas, aumentando la fuerza de la musculatura del suelo pélvico y la calidad de vida de los pacientes (17). Sin embargo, debemos resaltar que la realización de estos ejercicios debe de ser continuada en el tiempo para mantener los resultados $(17,18)$.

Las intervenciones y actividades planificadas para mejorar el sueño y la imagen corporal están avaladas por la comunidad científica (19-21).

Los profesionales de la salud deben ofrecer medidas preventivas adecuadas a los pacientes de alto riesgo (10). Para prevenir la aparición de complicaciones venosas se debe incidir en la realización de ejercicio físico regular, en el control del peso, en la dieta y en la aplicación de emolientes y barreras protectoras. Además, en pacientes con IVC y/o antecedentes de úlceras, las medias de compresión ayudan a la restauración del sistema venoso y reducen la aparición de complicaciones.

Por último, destacar que la planificación de cuidados debe de ser individualizada, adaptada a las características del paciente, de su enfermedad y de su entorno. De esta manera brindaremos unos cuidados enfermeros de calidad, enfocados al paciente desde una perspectiva biopsicosocial.

La principal limitación de este estudio es que, al tratarse de un caso individualizado, los resultados no pueden generalizarse.

\section{Conclusiones}

Una correcta planificación de cuidados contribuye a la mejora de la adherencia terapéutica del paciente, a conseguir una mejor adaptación a su proceso de cuidado y a reducir el impacto de la enfermedad sobre su imagen personal.

Por otro lado, las medidas higiénico-dietéticas (hidratación, higiene, hábitos adecuados, ejercicios de relajación y de fortalecimiento del suelo pélvico), junto con la compresión terapéutica, contribuyen a la mejora de los autocuidados y del manejo de la enfermedad.

\section{Conflicto de intereses: ninguno.}

Financiación: ninguna.

\section{Agradecimientos}

Especialmente queremos agradecer la participación de la paciente del caso, y su previa autorización para publicar los resultados y los cuidados otorgados.

\section{Bibliografía}

1. Otrante D.C.E, Quiñones- Castro M, Borrás- Migues M, Rodríguez- Villalonga L, \& Chirino- Día L. Insuficiencia venosa crónica y calidad de vida. Rev. Cub. Ang. Cir. Vasc. 2010; 11(1): 27-33.

2. Silva- Pupo M, del Risco- Turiño A, \& GonzálezMoya A. Caracterización de la insuficiencia venosa crónica profunda por reflujo valvular. AMC. 2014; 18(1): 30-41.

3. Farias- Dias T.Y, Fernandes- Costa I.K, MedeirosMelo M.D, Gomes Simões de Oliveira Torres S.M.S, Chaves- Maia E.M, \& de Vasconcelos Torres G. Evaluación de la calidad de vida de pacientes con y sin úlcera venosa. Rev Lat Am Enfermagem. 2014; 22(4):576-81.

4. Bellmunt S, Miquel C, Reina L, \& Lozano F. La insuficiencia venosa crónica en el Sistema Nacional de Salud. Diagnóstico, indicaciones quirúrgicas y priorización de listas de espera. Angiología. 2013; 65(2):61-71.

5. Gordon M. Manual de diagnósticos enfermeros. 10 edición. Madrid: Mosby; 2007.

6. Herman T.H. NANDA Internacional: Diagnósticos enfermeros: definiciones y clasificación. 2015-2017. Barcelona: Elsevier; 2015.

7. Johnson M., L., Mass M., Moorhead S., \& Swanson E. Clasificación de Resultados de Enfermería (NOC). 5á edición. Barcelona: Elsevier; 2014.

8. Bulechek, G., Butcher, H., Dochterman, J., \& Wagner, C. Clasificación de Intervenciones de Enfermería (NIC). 6a edición. Barcelona: Elsevier; 2014.

9. Córdoba-García R. Educación sanitaria en las enfermedades crónicas. Aten Primaria. 2003; 31:315-8.

10. Imbernón-Moya A, Ortiz-de Frutos FJ, SanjuanÁlvarez M, Portero-Sánchez I, Aguilar-Martínez A, Gallego-Valdés MÁ. Enfermedad venosa crónica. Clínica, clasificación, factores de riesgo, cuidados básicos, prevención, coste económico y pronóstico. Piel. 2018; 33(1):33-9.

11. Clark M. What are the differences between different compression materials? Journal of Wound Technology. Compression. 2010; (8), 6-64. Documento en internet. Disponible en:

http://www.tagungsmanagement.org/icc/images/st ories/PDF/publications_jwt.pdf

12. Gorgues- Zamora J. Adaptación de medias de compresión a medida. Offarm. 2011; 30: 76-8.

13. Raju S, Hollis K, \& Neglen P. Uso de medias de compresión en la enfermedad venosa crónica: grado de cumplimiento y eficacia. Ann Vasc Surg. 2007; 21(6):145-51. DOI: 10.1016/j.avsg.2007.07.014

14. Moratilla CA. Casos Clínicos Epaderm ${ }^{\circledR}$. Mölnlycke Health Care. 2016. Documento en Internet. Disponible en:

https://es.slideshare.net/carmenalbamoratilla/caso s-clinicos-epaderm-cream

15. Azcona L. Insuficiencia venosa. Prevención y tratamiento. Farm. Prof. 2008; 22:36-40. 
16. Carrasco- Carrasco E., \& Díaz- Sánchez S. Recomendaciones para el manejo de la Enfermedad Venosa Crónica en Atención Primaria. SEMERGEN. 2015.

17. González- Sánchez B., Rodríguez-Mansilla J., Toro García A. de, \& González López-Arza M.V. Eficacia del entrenamiento de la musculatura del suelo pélvico en incontinencia urinaria femenina. An. Sist. Sanit. Navar. 2014; 37(3), 381-400.

18. Carlos E, \& García B. Disfunción del piso pelviano en el adulto mayor. Rev. Med. Clin. Condes. 2013; 24(2): 196-201. Documento en internet. Disponible en: https://www.clinicalascondes.cl/Dev_CLC/media
/Imagenes/PDF\%20revista\%20médica/2013/2\%20 marzo/3-Dr.Garc-ia.pdf

19. Miró E., Cano- Lozano M., \& Buela- Casal G. Sueño y calidad de vida. Rev Colomb Psicol. 2005;14: 1127.

20. Corbella MJ. La alimentación y el sueño. Estrategias nutricionales para evitar el insomnio. Offarm. 2007; 26(5): 77-84.

21. Fernández-Bustos JG, González- Martí I., Contreras O., \& Cuevas R. Relationship between body image and physical self-concept in adolescent females. Rev Latinoam Psicol. 2014; 47 (1): 25-33 